\title{
One-dimensional infiltration equation based on Philip, Green, and Ampt models (El Kerma-Oran- Algeria)
}

BOUALLA Nabila ( $\square$ bouallanabila2021@gmail.com )

University of Science and Technology USTO-MB

\section{Research Article}

Keywords: Modeling, Infiltration, Discharge, Porous medium, Unsaturated soil

Posted Date: March 1st, 2022

DOI: https://doi.org/10.21203/rs.3.rs-1409076/v1

License: (c) (1) This work is licensed under a Creative Commons Attribution 4.0 International License.

Read Full License 


\title{
One-dimensional infiltration equation based on Philip, Green, and Ampt models (EI Kerma-Oran-Algeria)
}

\section{BOUALLA Nabila}

University of Science and Technology USTO-MB, Faculty of Architecture and Civil Engineering, Laboratory of Materials, Soils, and Thermal, B.P 150531000 Oran (Algeria).

Contact E-mail: bouallanabila2021@gmail.com, nabila.boualla@univ-usto.dz

\begin{abstract}
The control of water discharges in the El Kerma area is of paramount importance. These soils, which are generally rich in clay materials, are characterized by a (very) low permeability that leads to a rapid rise in pore pressure in the event of a massive influx of water. Thus, the prediction of the rate of water infiltration is essential for the prevention of flood risks.

It is mainly stormwater that must be feared. However, these waters cannot always be carried out in a network, because of the connection distance, or the insufficiency of its section in front of the extension of the waterproofed soils, which transport too fast high flows. Sanitation and urban development specialists are interested in the increase in impervious surfaces due to the development of urbanization that leads to excessive runoff flows during rainy periods. Their control is therefore much more delicate than that of wastewater, whose flows are generally low and spread over time.

The objective of this part is to study the infiltration regimes by using three models chosen for their simplicity (Philip, Green, and Ampt) and compare them to obtain the most effective model. Unfortunately, the result was not adequate since the infiltration time of the treated sample was short.
\end{abstract}

KEYWORDS: Modeling, Infiltration, Discharge, Porous medium, Unsaturated soil.

\section{Introduction}

Soil is a porous, dynamic, and open environment. It is the interface between the atmospheric system and the aquifer. Its peculiarity is that its water content varies depending on the depth. Two levels are depending on the level of water saturation: -an area saturated with water where water flows into the network of pores. -an area that is not saturated with water, where water is retained in the soil and has very little to no flow. Not all pores are filled and the ability of the water to drain is minimal [1], [2].

The study of flows in soil masses is part of a very broad field of research, consisting of the study of fluid movements in porous media. In the case of a mechanical application of soils and rocks, the porous medium studied is a natural soil mass (diffusion of pollutants, infiltration, flow, shrinkage of the water table, etc.). The circulation of a fluid through a porous medium, or more particularly, the water in the soil, can have major repercussions on the technical and geotechnical studies of the work in question.

Another aspect concerns the current problems of infiltration of pollutants generated by waste stored in public landfills; this leads to a risk of contamination of the water table. Often the compacted soils used in these sites are in a partially saturated state, which further complicates the problem because the hydraulic conductivity to unsaturated depends on the water content and the soil suction. 


\section{Water distribution in the soil during infiltration}

The distribution of water in the soil at a given time can be described by a water profile. The shape of the soil is related on the one hand to the physical properties of the soil and the other hand to highly variable weather conditions. Surface water infiltration causes a disturbance of the water profile that spreads deep as the process continues.

During infiltration, the water enters the soil and gradually descends downwards by establishing successive water content profiles (Figure 1).

The distribution of water in the soil generally depends on the size of the grain. The suction gradient, which was initially high, especially for dry soil, decreases during infiltration to unit values (Figure 1). This explains the decrease in infiltration speed over time.

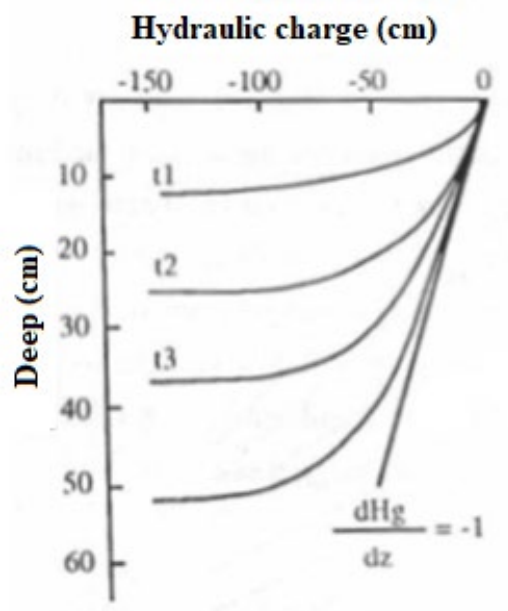

Figure 1 Hydraulic load profiles and water content profiles during infiltration into surface submerged soil [3]

Figure 2 shows the diagram of the storage components of an infiltration trench and associated water balance elements. The physical storage provided by an infiltration trench is mainly provided by its storage reservoir and the shallow depression storage created by the relatively low surface of the storage reservoir [4].

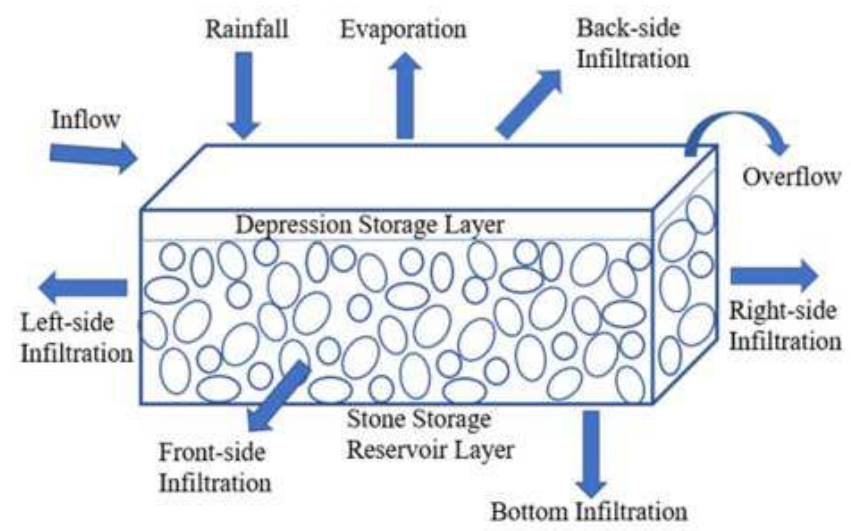

Figure 2 Schematic diagram of the storage layers and hydrologic components of an infiltration trench [4].

\section{Materials and methods}

\subsection{Isothermal Flow of Pure Water: Single-phase Approach}

A significant simplification of transfer equations can be achieved when considering the transfer of pure water in an isothermal and deformable medium with atmospheric air pressure. Let us 
first state the basic assumptions that will have to be satisfied for the equations established in the following to be verified.

Working hypotheses: -The flow is isothermal; - The liquid phase is continuous and consists of pure water, only the movement of the liquid phase will be considered; - The soil is inert, and its structure is non-deformable: * the solid phase is not dissolved in water; ${ }^{*}$ no production of material by a chemical reaction between the liquid phase and the solid phase; * no interaction between water and the solid phase (no swelling); -The air in the soil is subject to atmospheric pressure everywhere; -The term inertia is negligible and the generalized Darcy law is valid; The flow is one-dimensional.

These hypotheses thus amount, among other things, to neglecting the flow of air and that of water vapor.

a. Continuity equation: This equation, also called the mass conservation equation, establishes a proportionality between any local change and the associated flow difference (The balance of flows passing through the faces of an elementary volume of soil is also obtained by the difference of flows entering and leaving in the direction of the main axes). It is written in the following general form (Equation 1):

$\frac{\partial\left(\rho_{w} \theta\right)}{\partial t}=-\operatorname{div}\left(\rho_{w} q\right)$

If the density of the water is constant, the equation is written as follows equation 2 :

$\frac{\partial \theta}{\partial t}=\operatorname{div}(q)$

$\rho_{w}$ : density of water; $\theta$ : density of water in the medium; q: unit water flow (flow). This equation expresses the fact that the spatial variability of the flow corresponds to the temporal variability of the water content, i.e., temporal variability of the storage of water in a soil volume element.

b. Dynamic equation: The dynamic equation links the transfer flow to the force that generates it. By analogy with Darcy's law for flow in a saturated porous medium, this equation was called generalized Darcy's law in unsaturated flow conditions. This concept is only valid if the interaction at the water-air interface is weak. The general form of the dynamic equation is (Equation 3) [5]:

$q=-k_{w}(\theta) \operatorname{grad}(\varnothing)$

where, q: is the unit flow rate $(\mathrm{m} / \mathrm{s}), k_{w}(\theta)$ : the hydraulic conductivity coefficient $(\mathrm{m} / \mathrm{s}), \emptyset$ : the total potential.

c. Flow equation: This equation is obtained by combining the continuity equation and the dynamic equation. Various formulations are possible, depending on whether the various terms of this equation are expressed as functions of water content or suction. If the density water content is taken as the main variable, the unsaturated flow equation (Richards' equation) is written in equation 4 :

$\frac{\partial \theta}{\partial t}=\operatorname{div}\left(k_{w}(\theta) \operatorname{grad}(\varnothing)\right)$

Its application makes it possible to remedy the problem posed by the Fokker-Planck equation since suction varies continuously in the area of flow, passing from positive values in saturated zones to negative values in unsaturated zones (Vauclin, 1975) [6]

If, however, suction is considered as the main variable, this equation becomes equation 5 :

$c(\psi) \frac{\partial \psi}{\partial t}=\operatorname{div}\left(k_{w}(\psi) \operatorname{grad}(\varnothing)\right)$

Where: $c(\psi)=\frac{\partial \theta}{\partial \psi}$ is the capillary capacity given by the slope of the retention curve $\theta(\psi)$ at a particular value of suction. It expresses the variation of the water content per unit of variation of suction in the soil. These two expressions constitute the two forms of the Richards equation. To simplify the mathematical and experimental processing of unsaturated flow processes, it is often advantageous to transform flow equations into equations of forms analogous to that of 
diffusion and heat conduction. For this type of equation, there are defined solutions that can be used to the extent that boundary conditions to the process of water flow into the soil [7]. By introducing the diffusivity variable $\mathrm{D}(\theta)$ defined by equation 6 :

$\mathrm{D}(\theta)=\frac{k_{w}(\theta)}{c(\theta)}=k_{w}(\theta) \frac{\partial \psi}{\partial \theta}$

In this equation (general flow equation), the following equation (Equation 7 ) is obtained in the case of a vertical flow:

$\frac{\partial \theta}{\partial t}=\operatorname{div}\left(\mathrm{D}(\theta) \operatorname{grad} \theta+\frac{\partial k_{w}(\theta)}{\partial z}\right.$

From this equation, it can be said that water transfers to the unsaturated area depend on the hydrodynamic characteristics of the soil, namely the relationships between the suction and the water content of the soil and between the hydraulic conductivity and the water content. Knowledge of these two relationships is indispensable for the study of these transfers.

It is known as the non-linear Fokker-Planck equation. The use of this equation is limited for medium and low water contents. Indeed, in the range of high water contents, close to saturation, the diffusivity becomes indeterminate and tends towards infinity because the capillary capacity tends towards zero. This equation cannot, therefore, be used in the case where the analysis involves infiltration by submersion or coupling between infiltration and aquifer.

\subsection{Empirical models based on the fundamental equations of flow in a porous medium}

These models describe in a simplified way the movement of water in the soil, especially at the level of the humidification front and according to certain physical parameters. The best-known models are the Philip model and the Green and Ampt model.

a. Philip's Model (1957)[8]: Philip proposed a method of solving the vertical infiltration equation for certain initial conditions and limitations. This model introduces the concept of sorptivity which represents the capacity of a soil to absorb water when the flow occurs only under the action of the pressure gradient. Sorptivity is defined by the infiltrated blade I in horizontal flow. It depends on the initial and boundary conditions of the system. It is a function of the initial water contents of the soil and imposed on the surface.

The partial differential equation used to describe the flow of fluids in a one-dimensional vertical unsaturated soil is obtained by combining Darcy's generalized law with the conservation of mass equation. This leads to the Richards equation (Equation 8) [9], [10],[11] defined by :

$\frac{\partial \theta}{\partial t}+\frac{\partial}{\partial z}\left(-k_{w}\left(\frac{\partial h}{\partial z}+1\right)\right)=0$

Where $\mathrm{t}$ is time $(\mathrm{t}), \mathrm{z}$ is the soil depth taken positive downwards, $\theta$ is the volumetric water content, $\mathrm{h}$ is the soil water pressure which is negative for unsaturated flow, and $\mathrm{K}$ is the hydraulic conductivity, and the transport equation (Equation 9) to consider is:

$\frac{\partial \theta}{\partial t}=\frac{\partial}{\partial z}\left[D(\theta) \frac{\partial \theta}{\partial \theta}\right]+\frac{d k_{w}}{d \theta} \frac{\partial \theta}{\partial z}$

And the boundary conditions are: $\theta(z \leq 0 ; t=0)=\theta_{i} ; \theta(z=0 ; t>0)=\theta_{i} ; \theta(z \rightarrow \infty ; t>$ $0)=\theta_{i}$

Similar to the previous case of horizontal infiltration with vertical $\mathrm{D}$; there is no simple analytical solution for the transport equation (V-L) subject to boundary conditions.0r, Approximate solutions are needed. Philip (1957) demonstrated that an implicit solution (i.e., expressing $\mathrm{z}$ as a function of $(\theta)$ and $\mathrm{t}$ instead of $(\theta)$ as a function of $\mathrm{z}$ and $\mathrm{t}$ ) exists. This solution (Equation 10) is in the form of a series:

$z(\theta, t)=\sum_{i=1}^{\infty} g_{i}(\theta) t^{1 / 2}=g_{i}(\theta t) t^{\frac{1}{2}}+g_{2 i}(\theta) t$ 
With $g_{i}$ an auxiliary function that depends on the hydrodynamic properties of the soil. Let us take again the definition of the infiltrated water slide: Substitution in the two equations results in equation 11:

$$
I=\int_{\theta_{1}}^{\theta_{i}} z(\theta, t) d \theta \quad(10) \rightarrow I=\int_{1}^{\infty} f(\theta) t^{\frac{1}{2}}
$$

With $f_{1}$ :an auxiliary function that depends on $\mathrm{D}$ and $\mathrm{K}$., This solution should be simplified by considering only the first two terms of the series. The first term $f_{1}$ is called sorptivity which is denoted S. In the literature, we often put $f_{2}=\mathrm{A}$. So, the preceding equation is simplified as follows: $I=S \sqrt{t}+B t \quad$ (12) (equation of infiltration).

Which is Philip's famous undercover model. Remember, this equation is only applicable for a ground subject to boundary conditions, which corresponds to a ground flooded with the height of the water blade approximant zero.

The intensity of infiltration for Philip's model (Equation 12) is calculated as follows:

$$
I=\frac{d i}{d t}=\frac{1}{2} \frac{S}{\sqrt{t}}+B
$$

$\mathrm{B}$ : is a parameter having the dimensions of a velocity, which is of the order of $\frac{k_{s}}{3}<B<2 \frac{k_{s}}{3}$; is the conductivity of the soil to saturation. S: is the sorptivity that expresses the greater or lesser capacity of the soil to absorb water by capillarity. By derivation the infiltration speed is written in equation 13 :

$\frac{d I}{d t}=\frac{1}{2} S \sqrt{t}+B$

We will have equation 14 :

$$
\frac{I}{\sqrt{t}}=S+B \sqrt{t}
$$

The definition of S, according to Philip is as follows (Equation 15):

$$
S=\int_{\theta_{1}}^{\theta_{i}} \pi(\theta) d \theta
$$

With the Boltzmann transformation of the humectation front generated by capillary forces, and observed in a horizontal infiltration experiment.

The first term of the infiltration equation containing sorptivity is a measure of the capillary forces to moisten the soil. This term will be important at the beginning of the event but will decrease over time when the soil is moistened. This decrease is proportional to $\frac{1}{\sqrt{t}}$.

In the long term, when the soil becomes wet $(t>>0)$, the first term becomes negligible. It is the second term in the infiltration equation that will now determine infiltration. This second term is a measure of gravitational forces.

It can be deduced that sorptivity and B will be functions of the Hydrodynamic parameters of the soil. Several models are available to model the sorptivity of soil, the most exact model is perhaps that of Parlange (1971) [12], [13] (Equation 16):

$S=\sqrt{S\left(\theta_{s}+\theta-2 \theta_{i} D(\theta) d \theta\right.}$

An empirical approximation is that reported by Musy and Scoutter (1991) [14] (Equation 17):

$S=S_{0}\left(1-\frac{\theta_{i}}{\theta_{s}}\right)$

b. Model of Green and Ampt (1911) [15]: A simplified solution to the problem of infiltration is proposed by Green and Ampt (1911) [15]. In their approach, Green and Ampt put forward a series of hypotheses on the shape of the humectation profile, which led to a simplified physical solution. This solution lends itself more to the description of infiltration under constant load when a water table does not influence the water profile in the soil. This model is based on 
simplifying hypotheses involving a schematization of the infiltration process (Figure 3) (Equation 18).

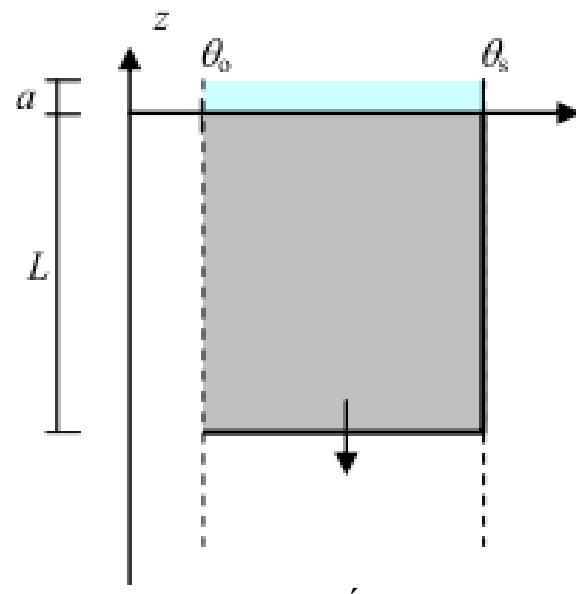

Figure 3 Sketch of Green-Ampts' infiltration process [16].

$I(t)=k_{s}\left(1+\frac{h_{0}-h_{f}}{Z_{f}(t)}\right)$

Where: $k_{s}$ : hydraulic conductivity at saturation $[\mathrm{mm} / \mathrm{h}] ; h_{0}$ : surface pressure load $[\mathrm{mm}] ; h_{f}$ : pressure load at the humidification front $[\mathrm{mm}] ; Z_{f}$ : depth reached by the humidification front [mm].

Saturated hydraulic conductivity describes the ability of saturated soil to drive free water. It is one of the most important soil characteristics to be determined when designing passive barriers, and is the key to understanding and calculating the flow conditions [17], [18], [19].

The model of Green and Ampt is based on simplifying assumptions that involve a schematization of the infiltration process. It is based on Darcy's law and includes the hydrodynamic parameters of the soil such as the hydraulic loads on the surface and at the level of the humidification front. The application of Darcy's (Equation 19) law allows writing:

$q=\frac{d I}{d t}=-k_{s} \frac{h_{f}-h_{0}-Z_{f}}{Z_{f}}$

One of the assumptions in the Green and Apmt model states that the water content of the transmission area is uniform. The cumulative infiltration $I(t)$ results from the product of the change in water content and the depth of the humidification front. This model is satisfactory when applied to the soil with a coarse texture. However, this method remains empirical since it requires the experimental determination of the value of the pressure load at the humidification front.

The existence of the wet zone extending to the humidification front allows us to write (Equation 20, 21, 22):

$I=Z_{f}\left(\theta_{s}-\theta_{i}\right)=Z_{f} \Delta \theta$

$\theta_{i}$ : initial volumetric water content, $\theta_{s}$ : saturation volumetric water content.

$\frac{d I}{d t}=-k_{s}\left(-1+\frac{\left(h_{f}-h_{0}\right) \Delta \theta}{I}\right)$

The integration of the equation with condition $I=0$ for $t=0$ gives:

$k_{s} t=I+\left(h_{f}-h_{0}\right) \Delta \theta \ln \left(1-\frac{I}{\left(h_{f}-h_{0}\right) \Delta \theta}\right)$

For relatively small times, infiltration I is small and the term of the equation $\frac{I}{\left(h_{f}-h_{0}\right) \Delta \theta}$ is much smaller than 1. By developing the term in Ln of the Taylor equation limited to the first two terms. We can write (Equation 23, 24, 25, 26, 27): 


$$
k_{s} t=\frac{I^{2}}{2\left(h_{f}-h_{0}\right) \Delta \theta}
$$

Where:

$$
I \approx \sqrt{\left(-2 k_{s}\left(h_{f}-h_{0}\right) \Delta \theta\right)} \sqrt{t}
$$

Either by identification with the original Philip law:

$$
I=S \sqrt{t} \text { (25) }
$$

We have:

$$
\begin{aligned}
& S \approx \sqrt{\left(-2 k_{s}\left(h_{f}-h_{0}\right) \Delta \theta\right)} \\
& S \approx i s 6)
\end{aligned}
$$

$\mathrm{P}$ being the slope of the adjustment line of $d I / d t$ according to $1 / I$. We therefore adjusted $d I / d t$ according to $1 / I$ :

$\frac{d I}{d t}=\frac{P}{I}+k_{S}$

However, As for the Philip adjustment, the starting assumptions of being not satisfied, we consider that we obtain a and b but not $\mathrm{S}$ and $k_{s}$.

\section{Results}

The procedure used was very primary: One tube of $3 \mathrm{~cm}$ of each sample was filled and $2 \mathrm{~cm}$ of water was added. Representative results are

shown in figures 4,5 , and 6 in which the relationships of infiltration rate versus cumulative infiltration are compared under the conditions of infiltration and rainfall infiltration.

a. Philip's MODEL: We initially chose to model the experimental $I(t)$ curves from an equation of this shape and by adjusting two parameters that we will call S and B. Indeed, if the heterogeneity of the soil texture and the non-uniformity place us, strictly, out of the conditions of application of this model.

Figure 4 presents the infiltrated volume over time estimated with both the proposed expressions. It can be observed the fairly good agreement between the infiltrated volume from the reposed general expression (Eq 14).

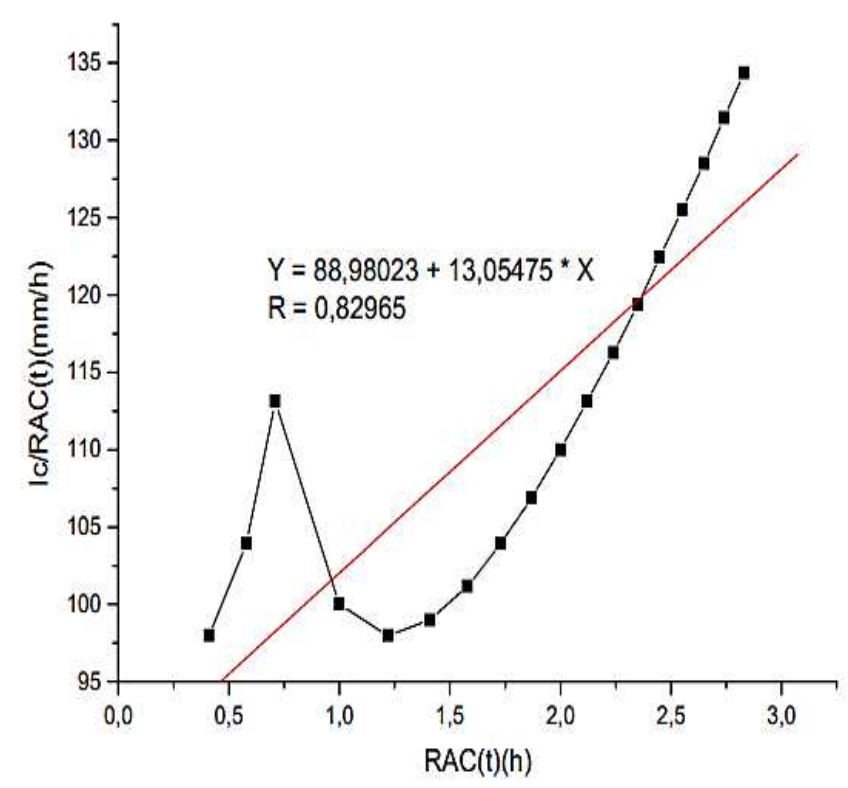

Figure 4 Infiltrated volume over time with the proposed Philip's model.

Based on the form of the equations. (11) and (14), the cumulative infiltration formula is relatively complex and cannot directly reflect the relationship between cumulative infiltration 
and wetting front depth, but the slope of the linear function I / t can be described using linear regression and equations [20].

The values of $\mathrm{S}$ and $\mathrm{B}$ are calculated by linear regression of $I / t$ on $\mathrm{t}$. According to the equation, we obtain: $\mathrm{S}$ being assimilated to the sorptivity of PHILIP, it is of the order of 13.055 . B being assimilated to hydraulic conductivity, it is of the order of $88.98 \mathrm{~mm} / \mathrm{h}$.

From the results obtained, it is noted that the values of sorptivity and conductivity depend in particular on the state of the environment studied and the load (quantity of water above the soil surface).

Moreover, because the solution of the water content is implicit and the change in water content is sudden in the transient phase, it is difficult to examine enough points to calculate the relative error in the small wet frontal distance, especially when the water content changes rapidly.

The numerical solution gave higher values than the approximate solution (Figure 5) below the intersection, in areas with low water content, the approximate solution gave higher values than the numerical solution. This indicates that at least during this initial range of soil water, the shape of the soil water profile was independent of the initial soil water content [20].

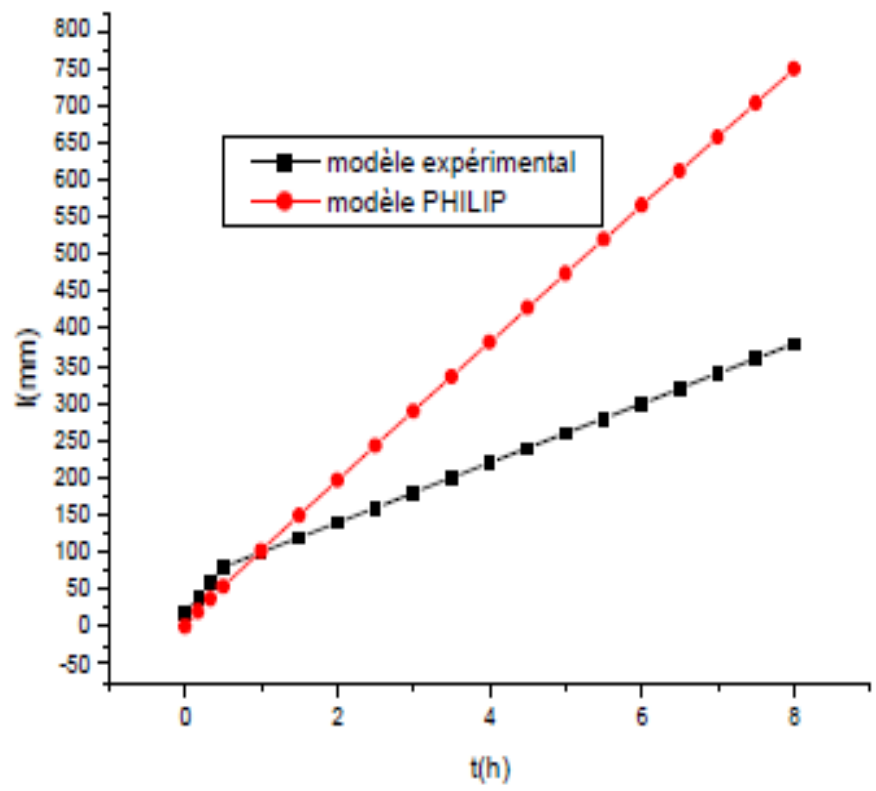

Figure 5a Comparison of the intensity of infiltration results of the numerical and approximate solutions calculated by Philip's model.

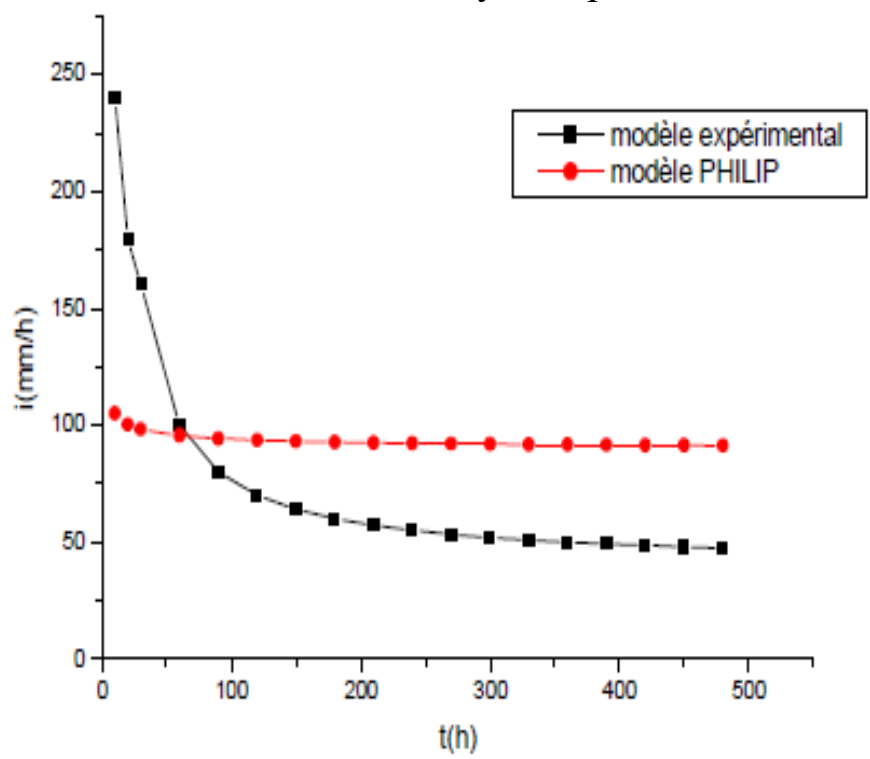


Figure 5b Comparison of the infiltration speed results of the numerical and approximate solutions calculated by Philip's model.

b. Green and Ampt model: The fit gives a correlation coefficient: $\mathrm{R}^{2}=0.98$

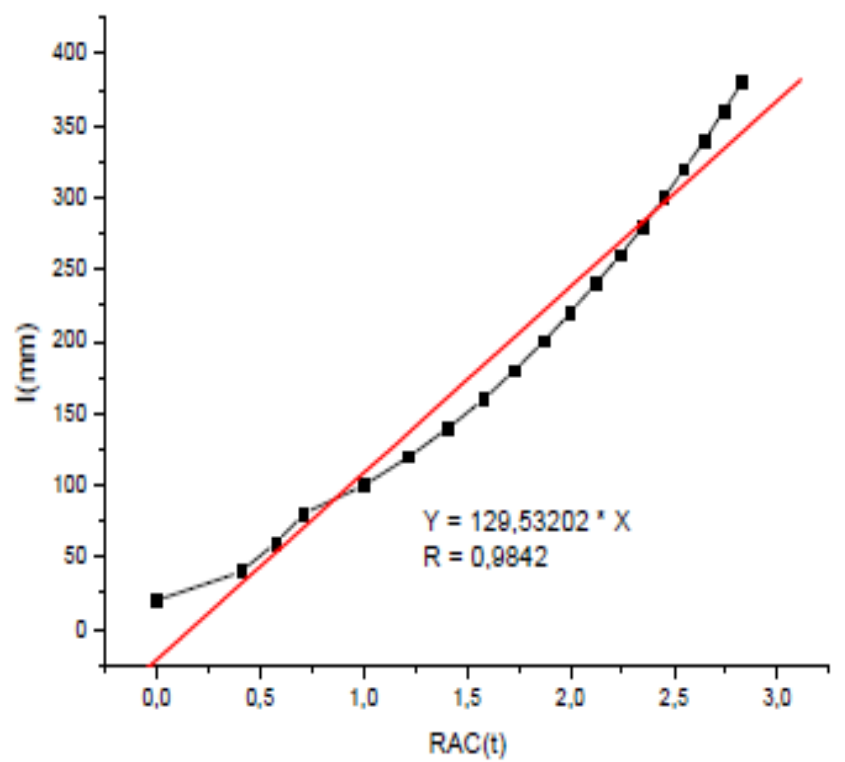

Figure 6a Infiltrated volume over time with the proposed Green and Ampt model.

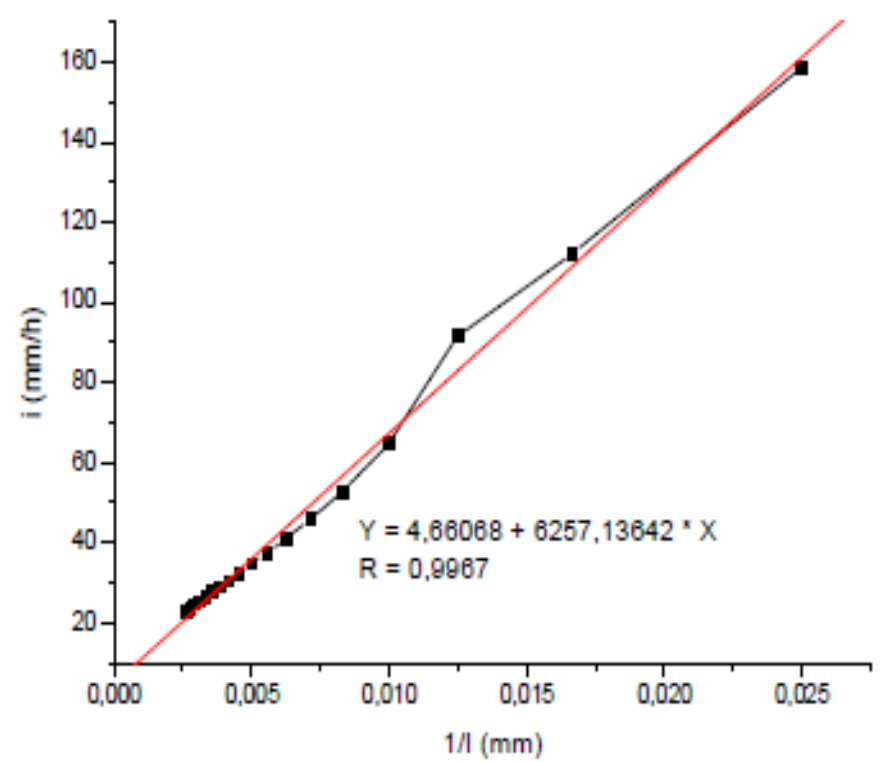

Figure 6b Infiltrated volume over adjustment line of $d I / d t$ according to $1 / I$ with the proposed Green and Ampt model.

Model $I=f(t)$ gives a less satisfactory fit. 


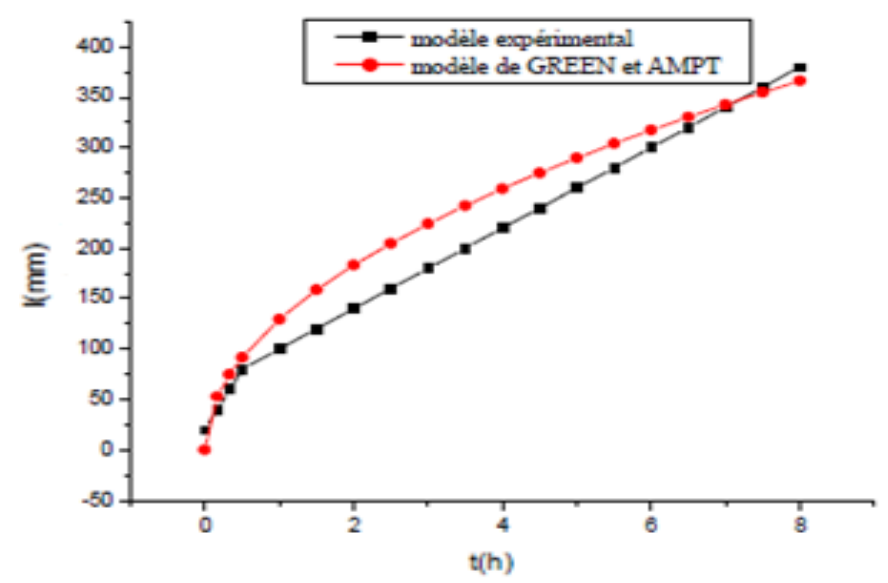

Figure 6c Comparison of the intensity of infiltration results of the numerical and approximate solutions calculated by Green and Ampt model.

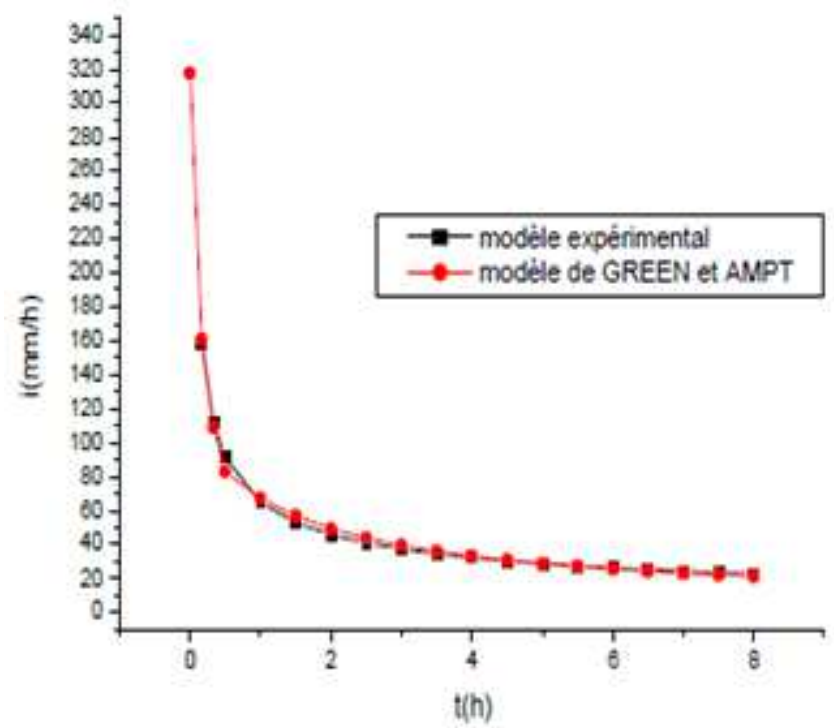

Figure 6d Comparison of the infiltration speed results of the numerical and approximate solutions calculated by Green and Ampt model.

The calculated values adjust with the observed values. According to the results obtained, the Soptivity (S) values calculated differently according to S1 and S2 are identical. S2 is greater than S1.

As seen from equation (18), the infiltration rate obtained here can be expressed as a linear function of the depth of the reverse wetting front (Figure 6). The intersection of the vertical axis and the linear function describing the relationship between the infiltration rate and the inverse depth of the wetting front is equal to the saturated hydraulic conductivity based on soil physics. This indicates that at least during this initial range of soil water, the shape of the soil water profile was independent of the initial soil water content [20].

Nevertheless, these differences in cumulative infiltration will decrease with time and rainfall intensity (Figure 5, 6).

The run-on particularly affects infiltration and runoff on hydraulically heterogeneous surfaces and is the main mechanism for the scale effect in hortonian rainfall processes [21], [22].

We know from previous work [23]. That the Green Ampt solution tends to overestimate infiltration at intermediate stages while it adapts better to early or long times. As a result of the previous arguments, we can deduce that the main problem in the simulation of the infiltration without gravity must be the heterogeneity of the soil (, not addressed in this article [16]. 


\section{Conclusion}

In this article, a simple exact solution of the one-dimensional dimension. The transient and nonlinear Richards equation is presented. These exact solutions can be used to model problems with time-dependent or constant boundary conditions (water content and flow conditions) [10]. Such hydraulic functions are frequently used in the literature and may be used to model real systems.

Several models were proposed to describe the infiltration curve. Most of these models are aimed at estimating the values of the sportiness of the diffusivity and permeability of the soil [8].

The tests were carried out to allow the estimation of these parameters.

The introduction of generalized Darcy's Law provides the equation for water transfer in unsaturated porous media. The resolution of this equation gives the Spatio-temporal distribution of moisture in porous media. This resolution is possible as soon as the hydraulic conductivity function $\mathrm{K}$ ( is known in the range of variation of the water contents. Empirical models or measurements can obtain this hydraulic conductivity. For each model K (a relationship between suction and volumetric water content has been associated.

The study presented focuses on the analysis and interpretation of laboratory water infiltration tests on unsaturated soil to provide some insight into water transfer. These results show that the infiltration of a liquid depends not only on the type of soil but also on the type of liquid. The three models tested give satisfactory results. Sorptivity according to the PHILIP model is very different from S2 of Green and Ampt.

The Green and Ampt model gives very good adjustments in terms of cumulative infiltration compared to the Philip models. Finally, the flows in porous media are made by complex and tortuous paths through a series of interconnected pores and of different sizes and shapes. As a general rule, permeability in a porous medium depends on:

- Pore volume (function of density and saturation); - Pore size distribution (internal pore structure). The composition includes the mechanical, physical, and chemical properties of the grains forming the framework of the soil sample. These properties are important for determining the ranges and boundaries of the geotechnical properties of soils, and in particular for permeability.

For example, the shape and distribution of the size of the grains are essential in determining the density, and more importantly for the internal structure (distribution of the size of the voids), which is also obtained, in the function of the water content. The size and shape of the grains depending on their mineralogy. The composition of soil plays an important role in determining the reactions (in terms of structural variation) in contact with the different pore waters.

Perspectives: -Vary the load -Spatial variability only in-depth -Influence of grain size -Use other types of liquid.

\section{Declaration of competing interest}

I declare that I have no significant competing interests including financial or non-financial, professional, or personal interests interfering with the full and objective presentation of the work described in this manuscript.

\section{References}

[1] P.G.Toledo, R.A.Novy, H.T.Davis, L.E.Scriven, "Hydraulic conductivity of porous media at low water content,"Soil Sci. Soc. Am. J., vol. 54, pp.673-679, 1990.

[2] S.J.Van der Hoven, D.K.Solomon, G.R.Moline, "Modeling unsaturated flow and transport in the saprolite of fracturted sedimentary rocks: Effects of periodic wetting and drying,". Water Resour. Res., vol. 39, pp. 1186-1195, 2003. 
[3] D.Hillel, "L'eau et le sol, principes et processus physiques," $2^{\text {nd }}$ ed, Bruxelles: Academia, $288,1988$.

[4] J.Wang, Y.Guo, "Dynamic water balance of infiltration-based stormwater best management practices,"Journal of Hydrology, vol. 589, no. 125174, 2020. https://doi.org/10.1016/j.jhydrol.2020.125174.

[5] D.G.Fredlund, A.Xing, S.Huang, "Predicting the permeability function for unsaturated soils using the soil-water characteristic curve," Can. Geotech. Journal, vol. 31, pp. 533-546, 1994.

[6] M.Vauclin, J. Imbernon, G.Vachaud, “Analyse comparative des différentes méthodes de détermination de la conductivité hydraulique des sols non saturés de la zone centre-nord du Sénégal,"L'Agronomie Tropicale, vol. 38, no. 3, pp.186-197, 1983.

[7] E. C. Childs and N. Collis-George, "The Permeability of Porous Materials Proceedings of the Royal Society of London," Series A, Mathematical and Physical Sciences, vol. 201, no. 1066 , pp. 392-405, 1950.

[8] J.R.Philip, "The theory of infiltration: 1. The infiltration equation and its solution,"Soil Science, vol. 83, pp. 345-357, 1957.

[9] L.A.Richard, "Capillary conduction of liquids through porous medium," Physics, vol. 1, pp. 318-333, 1931.

[10] M.Hayek, "An exact explicit solution for one-dimensional, transient, nonlinear Richards' equation for modeling infiltration with special hydraulic functions," Journal of Hydrology, vol. 35, pp. 662-670, 2016. http://dx.doi.org/10.1016/j.jhydrol.2016.02.021.

[11] N.Amraoui, "L'étude de l'infiltration dans les sols fins non saturés," Sciences Thesis INP Lorraine, 1996.

[12] J. Y. Parlange, "Theory of water-movement in soils: I. One-dimensional absorption," Soil science, vol. 111, no. 2, pp.134-137, 1971.

[13] J. Y.Parlange, "Theory of water-movement in soils: 2. One-dimensional infiltration," Soil Science, vol. 111, no 3,pp. 170-174, 1971.

[14] A.Musy, M.Soutter, "Physique du sol,"Lausanne, Pr. polytechniques et universitaires romandes, 1991.

[15] W.H.Green, G.A.Ampt, "Studies on soil physics: I. the flow of air and water through soils," J. Agric. Sci., vol. 4, pp. 1-24, 1911.

[16] A.Del Vigo, S.Zubelzu, L.Juana, "Infiltration models and soil characterisation for hemispherical and disc sources based on Green-Ampt assumptions," Journal of Hydrology, vol. 595, no, 125966, 2021. https://doi.org/10.1016/j.jhydrol.2021.125966.

[17] P. V.Sivapullaiah, A.Sridharan, ,V. K. Stalin, "Hydraulic conductivity of bentonite sand mixtures," Can. Geotech. J, vol. 37, pp. 406-413, 2000.

[18]B.Bussière, "Colloquium 2004: Hydrogeotechnical properties of hard rock tailings from metal mines and emerging geoenvironmental disposai approaches," Canadian Geotechnical Journal, vol. 44, pp.1019-1052. 2007.

[19]A. Demdoum, "Comportement hydrique et mécanique d'un mélange de BentoniteSableCalcaire-Tuf: Application à laconception des Installations de Stockage des Déchets (ISD) ," Thesis UNIVERSITy LAGHOUAT, Algeria, 2019.

[20] L.Su, J.Wang, X.Qin, Q.Wang, "Approximate solution of a one-dimensional soil water infiltration equation based on the Brooks-Corey model," Geoderma, vol. 297, pp. 28-37, 2017. http://dx.doi.org/10.1016/j.geoderma.2017.02.026.

[21] I.Chen, S.Sela, T.Svoray, S.Assouline, "The role of soil-surface sealing, microtopographe, and vegetation patches in rainfall-runoff processes in semiarid areas," Water Resour. Res., vol. 49, no 9, pp. 5585-5599, 2013. 
[22] I.Chen, S.Sela, T.Svoray, S.Assouline, "Scale dependence of Hortonian rainfall-runoff processes in a semiarid environement," Water Resour. Res., vol. 52, no 7, pp. 5146-5166, 2016.

[23] B.Sayah, M.Gil-Rodriguez, L.Juana, "Development of one-dimensional solutions for water infiltration. Analysis and parameters estimation,"J. Hydrol, vol. 535, pp. 226-234, 2016. 